\title{
Software Development based Implementation and Example Analysis of Computer Assisted English Learning System
}

\author{
LI Xiaoqin ${ }^{1, a}$ \\ ${ }^{1}$ Hubei University of Technology, Wuhan 430068, China \\ a Lisy1996@126.com
}

Keywords: Software Development; Example Analysis; Computer Assisted; English Learning.

\begin{abstract}
With the rapid development of software development technique and self-learning methodology, the computer assisted learning pattern is being accepted by more and more students. In this paper, we conduct research on the design and implementation of intelligent computer assisted English learning system. The rapid development of modern information technology and the bursting popularization of high-tech, people's work and life are more and more inseparable from the information. Our system uses the machine learning algorithm to understand the meaning of the students automatically. The performance is acceptable and satisfactory.
\end{abstract}

\section{Introduction}

Recent progress of computer and network technologies has encouraged the development of web-based learning environments in which students can deal with their learning activities without being limited by location and time. The think of college English should begin from the general English gradually turned to professional English. College English teaching orientation should be academic English. Academic English is divided into two categories: general academic English and English for special purposes. The former has academic written communication and oral communication curriculum, such as the latter have engineering English, financial English, software English and legal English courses, mainly to help them familiar with the vocabulary characteristics and syntactic structure and textual features. Both are for students in English major study prepared for the language and the content and learning skills. The rapid development of modern information technology and the popularization of high-tech, people's work and life are more and more inseparable from the information. Therefore shows the importance of interactive media is growing up. In modern times is the network technology rapid development of the information age. The use of the Internet for network teaching is the development trend of modern education. Biggest advantage lies in using it to computer network resource sharing. Use the interactive media, not only changed the way of learning and greatly reduce the cost of learning especially for the application of interactive media in the school.

The use of the Internet for network teaching and the development trend of modern education as a discipline and high speed information transmission can guarantee the implementation of the teaching get quick. Online teaching is really beyond time and space that anyone can learn at any time and place, students can also according to your own level. Interest and the time to choose the teaching content of study. The advantage of this method is evident and significant [1-3].

In this paper, we conduct research on the design and implementation of intelligent computer assisted English learning system. The current online and computer based learning systems are suffering from the following drawbacks. (1) Dynamic interaction function is not strong and mostly in the form of teaching materials piled up. Students just passively accept the teaching content and cannot be found actually involved in the system, create a type of active learning, it is difficult to reach the expected effect of learning. (2) System is lower intelligence. Not according to the current situation of students to provide appropriate, intelligent interaction interface, mobilize the students' interest. (3) System testing and evaluation function is not strong. Existing online testing system is put in fixed on a web page more fixed exam and cannot be automatically selected topic according to the 
requirements of students and the learning situation. Set up the remote online learning system based on Internet, not only can provide students a more efficient, more diversified, make people more likely to accept, but cheaper way of learning, can also to our country current education resources shortage and the contradiction between education demand growing provides an effective relief, it will drive the deep reform of education means and ways of learning [4-5]. Therefore, our research is meaningful and helpful. In the following sections, we will implement the core algorithms and code structure demo.

\section{Design and Implementation of the System}

The Intelligent System Algorithm Description. As mentioned, our target is to build up the intelligent learning system and it is more than necessary for us to conduct some theoretical analysis on the intelligent algorithm. Existing online learning system is limited to students to study hard, no interaction which is also impossible to knowledge to understand right and wrong and completely is the behavior of the individual, if the agent technology for online learning system and this way of learning will become more flexible and more easily accepted by people. To cope with this problem, a self-adjusting virtual tutoring assistant system is proposed in this paper. This system is able to answer students' questions automatically based on the training cases given by the teacher. Improved definition agent on the basis of the characteristics of weak definition includes the characteristics of human emotion [6]. Agent is an entity capable of autonomy and the entity is a support system by software. This software to the target driven, social learning behavior, respond actively to the change of environment, perform specific tasks. In the formula 1 3 we show the prior intelligent theory.

$$
F N\left(K(Q), K\left(D_{q}\right)\right)=\sum_{i=1}^{m 1} \sum_{j=1}^{m 2}\left(\text { equals }\left(K_{i}(Q), K_{j}\left(D_{q}\right)\right) \times w\left(K_{j}\left(D_{q}\right)\right)\right)
$$

To ensure the best-fit document to the questions submitted by the student can be retrieved, a set of training cases is collected to adjust the weights of the document keywords.

$$
Q=\left(\sum_{i}^{n} e_{i i}-\sum_{i}^{n} a_{i} b_{i}\right) /\left(1-\sum_{i}^{n} a_{i} b_{i}\right)
$$

In the analysis of software structures, this metric is useful because in many cases the definition of modules is given by means of programming constructs. It is used to study the feedback. Members can make your own or from other ways to collect to tutorial published to the web site for other users to share, can also publish tutorials about themselves. The user can through the message board giving their views and to communicate with others.

$$
\operatorname{rep}(i)=\sum_{x} \operatorname{rep}(x, i), \Delta x \in \operatorname{subH}(i)
$$

Cross compile technical support cross-platform mobile learning system powerful, real life situation and positive interaction, suitable for all basic functions of mobile learning, especially suitable for image rich game of augmented reality, explore and study function of mobile learning [5]. In the following sections we will finalize the process of the system development.

\section{The System Structure Description.}

A mature platform for the students should be able to realize the online or offline learning, the demand of the use of fragmented time learning and the students can learn anytime and anywhere through a mobile operator, development technology is mainly used here as the follows [7-8]. (1) Cross-compilation technology. Cross-compilation technology is a kind of host and target machine can be separation technology, cross-compilation is used on the host platform chain generated on the target machine can perform file, here we are using the JavaScript language, application using the API function to build. (2) Web app technique. Commonly used to run on a mobile browser technology is based on the mobile web app, it can is a kind of cross platform technology. We usually use HTML, CSS or JavaScript to organization development and application, in HTML and CSS advanced functionality using embedded SQL database, local storage, animation, network socket and video 
playback, these are consistent with the local APP on mobile web applications. (3) Hybrid mobile web app. Mixed mobile web app technology is a kind of local app embedded in one of the mobile browser technology. It can be done by custom connection API for communication between JavaScript web applications and web pages are usually stored in the local cache which can improve the access speed to establish a data connection. Mixed mobile web app has a good advantage of user interaction, and can realize cross-platform development, provides broad support for different terminals, can increase by mobile development is insufficient, because the function of mobile phone browser itself is insufficient, can't use standard elements within the network and a hybrid mobile web app technology, mobile phones without reconstruction resources content, it can be used to realize cross-platform. The following figure shows the pattern.

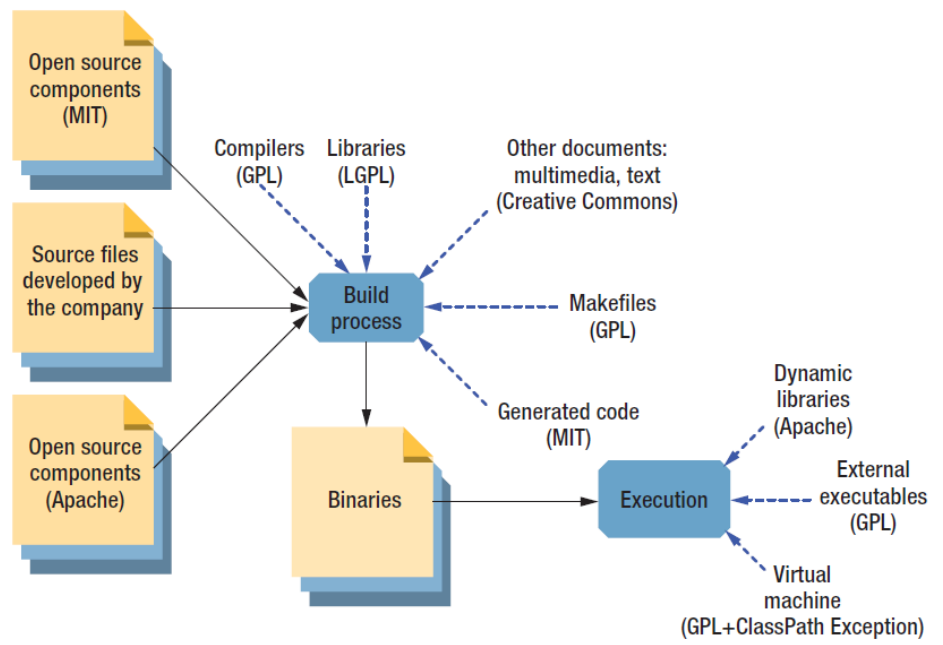

Fig. 1The System Structure Description for the Proposed System

\section{The Detail Implementation of the System.}

In the system, the following functions should be added and included. Media interactive learning system is mainly composed of message board module, the tutorial to retrieve module, release and management course module and we can view the tutorial module, background management module page. Users can download resources problems through the message board feedback. In the process of learning tutorial don't understand the problems can be through the message board to communicate feedback, etc. The platform structure is shown below.

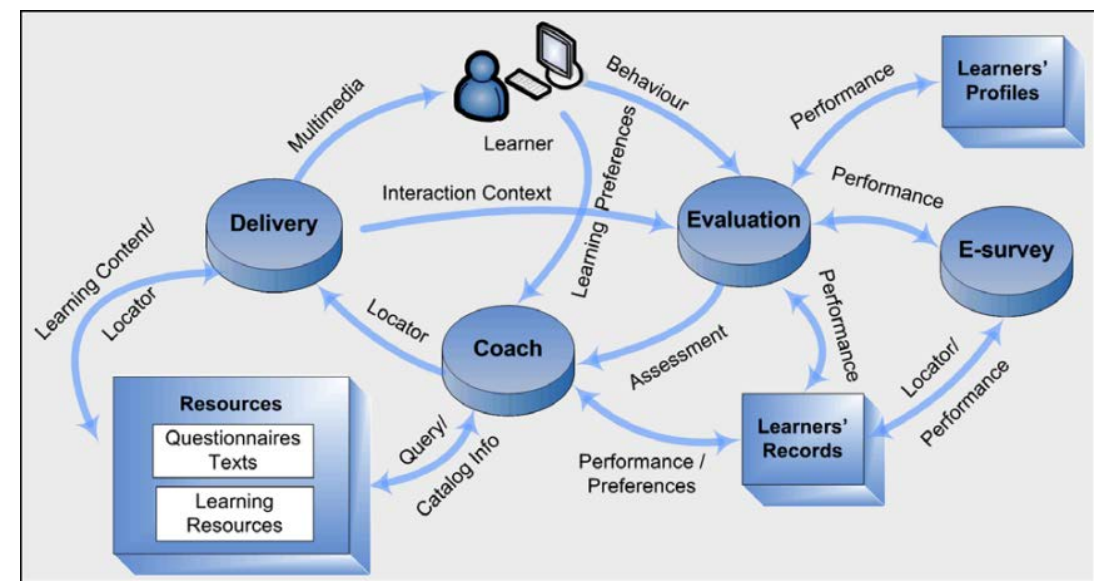

Fig. 2The Platform Structure and the Software Structure

This model will help to accelerate the access speed, the client using the browser on the Internet, no longer need to install different customers for different clients program, it can provide uniform 
environment for complex distributed applications. Middle Agent layer is all kinds of Agent, responsible for communication client and server, the server needs to run, such as HTTP and FTP agreement to aid communication. Ask agent layer between various agents also to be able to communicate with each other, to coordinate work. Students' agent will use the user and basic information from the user data table to retrieve the user's basic information. If a student is not the first time login, historical records by the agent to give the students in the past record, as students continue to learn the reference. Students according to interface, choose their own learning, students at the same time the agent will receive the information transmitted to management agent, by the management agent offer learning advice to students, and judgment with which the agent contact, browse the learning agent, exam answer the agent or the agent. Concrete study content, practice or test and the problems solutions were studied by browsing. Browse the learning agent, the content of the exam answer agent, the agent to provide again by the agent feedback to students. Students agent to collect the student's answer as a result, back to evaluate the test agent, the test agent will evaluation results to the management agent, agent management according to the evaluation results, provide further study suggest. The third layer is data layer which is used to hold all kinds of data resources and the knowledge base. The core code organization is shown in the following description.

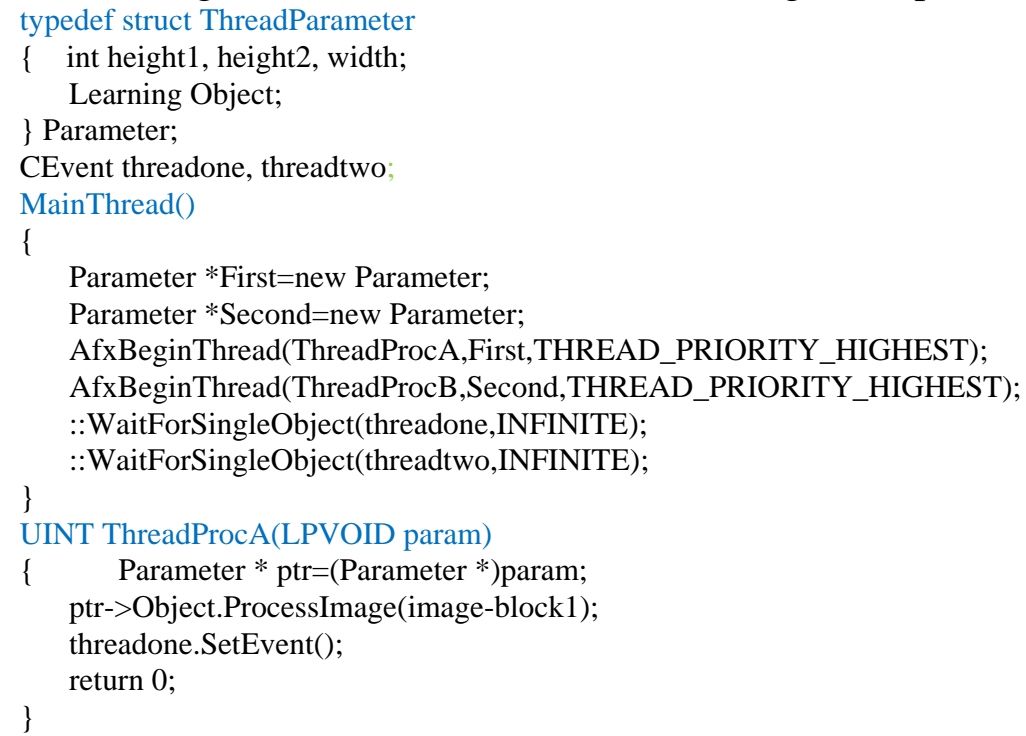

\section{Conclusion and Summary}

The advent of the computer to a large extent changed our traditional life and work of the party, the computer is applied to the teaching has is commonplace. With the development and the progress of science and technology, ushered in the era of knowledge explosion, knowledge update faster and faster. The contemporary high school students must be develop into lifelong learners to adapt to the knowledge explosion era. So the independent learning ability for the contemporary high school students is becoming more and more important. Computer aided English teaching for the majority of teachers and students provide the real text, images, audio, and video teaching resources, etc. The characteristics of computer assisted English teaching feedback learning results make it possible to students' autonomous learning.

In this paper, we conduct research on the design and implementation of intelligent computer assisted English learning system. The use of the Internet for network teaching is the development trend of modern education. Biggest advantage lies in using it to computer network resource sharing and the use the interactive media not only changed the way of learning and greatly reduce the cost of learning especially for the application of interactive media in the school. Our method adopts the basic concepts and principles of intelligent system. With the assistance of the machine learning algorithms and software development techniques, we built up the system successfully. Our future research will be focused on the interactive learning system which will be more vivid. 


\section{References}

[1] Ferrer, L., Bratt, H., Richey, C., Franco, H., Abrash, V., \& Precoda, K. (2015). Classification of lexical stress using spectral and prosodic features for computer-assisted language learning systems. Speech Communication, 69, 31-45.

[2] Corporation, H. P. (2015). Comparative analysis of classifiers for developing an adaptive computer-assisted eeg analysis system for diagnosing epilepsy.. Biomed Research International.

[3] Wu, W. H., Wu, Y. C. J., Chen, C. Y., Kao, H. Y., Lin, C. H., \& Huang, S. H. (2012). Review of trends from mobile learning studies: A meta-analysis.Computers \& Education, 59(2), 817-827.

[4] Huang, Y. M., Huang, Y. M., Huang, S. H., \& Lin, Y. T. (2012). A ubiquitous English vocabulary learning system: Evidence of active/passive attitudes vs. usefulness/ease-of-use. Computers \& Education, 58(1), 273-282.

[5] Wu, L. Y., \& Liu, C. (2015). Supporting seamless learning across individual, collaborative, and community-based learning in one-to-one learning age. Seamless Learning in the Age of Mobile Connectivity.

[6] Liao, Y., Huang, Y., Chen, H., \& Huang, S. (2015). Exploring the antecedents of collaborative learning performance over social networking sites in a ubiquitous learning context. Computers in Human Behavior, 313-323.

[7] Chen, C. M., \& Li, Y. L. (2010). Personalised context-aware ubiquitous learning system for supporting effective English vocabulary learning. Interactive Learning Environments, 18(4), 341-364.

[8] Ke, F. (2009). A qualitative meta-analysis of computer games as learning tools.Handbook of research on effective electronic gaming in education, 1, 1-32. 